\title{
ТОЧНЫЕ РЕШЕНИЯ ОБРАТНОЙ ЗАДАЧИ ОПТИМАЛЬНОЙ СТАБИЛИЗАЦИИ ДЛЯ СИСТЕМ С ПОСЛЕДЕЙСТВИЕМ НЕЙТРАЛЬНОГО ТИПА
}

\author{
Ю. Ф. Долгий
}

\begin{abstract}
Рассматривается задача оптимальной стабилизации для систем дифференциальных уравнений с последействием нейтрального типа. Для упрощения представления непрерывного квадратичного функционала используется изоморфизм функциональных пространств. Приведена постановка задачи оптимальной стабилизации в функциональном пространстве состояний со специальной метрикой. Описана постановка обратной задачи оптимальной стабилизации. Она связана с восстановлением системы, обладающей заданным представлением оптимального стабилизирующего управления. Получены достаточные условия разрешимости обратной задачи. Указаны условия, при выполнении которых обратная задача допускает аналитические решения. Предложен метод для нахождения точных решений этой задачи. Для систем дифференциальных уравнений с последействием запаздывающего типа точные решения обратной задачи получены раньше. Приведен пример точного решения обратной задачи для системы дифференциальных уравнений с последействием нейтрального типа.
\end{abstract}

Ключевые слова: дифференциальные уравнения с последействием нейтрального типа, оптимальная стабилизация, уравнение Риккати.

Yu. F. Dolgii. Exact solutions of an inverse optimal stabilization problem for systems with aftereffect of neutral type.

An optimal stabilization problem is considered for systems of differential equations with aftereffect of neutral type. To simplify the representation of a continuous quadratic functional, an isomorphism of functional spaces is used. The optimal stabilization problem is formulated in a functional space of states with a special metric. A statement of the inverse optimal stabilization problem is presented; this statement is related to the recovery of a system with a given representation of an optimal stabilizing control. Sufficient conditions for the solvability of the inverse problem are obtained, and conditions under which the inverse problem admits analytical solutions are specified. A method for finding exact solutions to this problem is proposed. For systems of differential equations with delay-type aftereffect, exact solutions of the inverse problem were obtained earlier. An example of the exact solution of the inverse problem is given for a system of differential equations with aftereffect of neutral type.

Keywords: differential equations with aftereffect of neutral type, optimal stabilization, Riccati equation.

MSC: 34K06, 34K20, 34K30

DOI: $10.21538 / 0134-4889-2019-25-1-35-44$

\section{Введение}

Для решения задачи оптимальной стабилизации линейной системы дифференциальных уравнений с последействием запаздывающего типа Н. Н. Красовский разработал метод квадратичных функционалов [1]. Оптимальное стабилизирующее управление единственным образом определяется коэффициентами квадратичного функционала, нахождение которых связано с решением операторного уравнение Риккати [2], порождающего систему определяющих уравнений $[3 ; 4]$. Этот подход получил развитие в работах [5;6]. При решении задач оптимальной стабилизации линейных систем дифференциальных уравнений с последействием нейтрального типа также применялся метод квадратичных функционалов [7;8]. В работе [8] предложена новая постановка задачи оптимальной стабилизации этих уравнений, которая позволяет использовать более простые представления квадратичных функционалов. Получена 
система определяющих уравнений для нахождения оптимального стабилизирующего управления. Исследование этой системы приводит к краевой задаче для нелинейного функциональнодифференциального уравнения нейтрального типа, решение которой определяет представление оптимального стабилизирующего управления для автономной линейной системы дифференциальных уравнений с последействием нейтрального типа. Проблема нахождения точных аналитических решений этой краевой задачи является достаточно сложной. В настоящей работе решается обратная задача оптимальной стабилизации, связанная с восстановлением системы обладающей заданным оптимальным стабилизирующим управлением.

\section{1. Задача оптимальной стабилизации}

\section{в функциональном пространстве состояний}

Объект управления описывается автономной линейной системой дифференциальных уравнений с последействием нейтрального типа

$$
\frac{d x(t)}{d t}=\int_{-r}^{0}[d \eta(\vartheta)] x(t+\vartheta)+\int_{-r}^{0}[d \mu(\vartheta)] \frac{d x(t+\vartheta)}{d \vartheta}+B u, \quad t \in \mathbb{R}^{+}=(0,+\infty) .
$$

Здесь $x:[-r,+\infty) \rightarrow \mathbb{R}^{n}, r>0 ; u \in \mathbb{R}^{m}$ - управление, $B-$ постоянная матрица; матричнозначные функции $\eta, \mu$ имеют ограниченные вариации на $[-r, 0], \eta(0)=0, \mu(0)=\mu(-0)=0$, $\operatorname{det} \eta(-r) \neq 0$.

Требуется найти управление, формируемое по принципу обратной связи, которое обеспечивает устойчивую работу системы (1.1) и минимизирует заданный критерий качества переходных процессов

$$
J=\int_{0}^{+\infty}\left(x^{\top}(t) C_{x} x(t)+u^{\top}(t) C_{u} u(t)\right) d t
$$

где $C_{x}$ и $C_{u}$ - положительно определенные матрицы.

При описании задачи оптимальной стабилизации в функциональном пространстве состояний традиционно используют пространство функций $\mathbb{H}^{1}=\mathbb{W}_{2}^{1}\left([-r, 0], \mathbb{R}^{n}\right)[4 ; 7]$. В работе [8] в этом пространстве вводится скалярное произведение

$$
\langle\mathbf{x}, \mathbf{y}\rangle_{\mathbb{H}^{1}}=\mathbf{y}^{\top}(0) \mathbf{x}(0)+\int_{-r}^{-0} \mathbf{y}^{\prime \top}(\vartheta) \mathbf{x}^{\prime}(\vartheta) d \vartheta, \quad \mathbf{x}, \mathbf{y} \in \mathbb{H}^{1}
$$

Использование этой метрики позволяет задачу оптимальной стабилизации для системы (1.1) с критерием качества (1.2) заменить задачей оптимальной стабилизации в пространстве $\mathbb{H}=$ $\mathbb{L}_{2}\left([-r, 0), \mathbb{R}^{n}\right) \times \mathbb{R}^{n}$ со скалярным произведением

$$
\langle\mathbf{x}, \mathbf{y}\rangle_{H}=\mathbf{y}^{\top}(0) \mathbf{x}(0)+\int_{-r}^{-0} \mathbf{y}^{\top}(\vartheta) \mathbf{x}(\vartheta) d \vartheta, \quad \mathbf{x}, \mathbf{y} \in \mathbb{H}
$$

Здесь при определении элемента $\mathbf{x} \in \mathbb{H}$ ему ставится в соответствие пара элементов $\left(\mathbf{x}_{1}(\cdot), \mathbf{x}_{2}\right)$, где $\mathbf{x}_{1}(\cdot) \in \mathbb{L}_{2}\left([-r, 0), \mathbb{R}^{n}\right), \mathbf{x}_{2} \in \mathbb{R}^{n}$ и полагается, что $\mathbf{x}_{1}(\vartheta)=\mathbf{x}(\vartheta)$ при $\vartheta \in[-r, 0), \mathbf{x}_{2}=\mathbf{x}(0)$.

При рассматриваемой замене используется изометрический изоморфизм $\pi$ пространств $\mathbb{H}^{1}$ и $\mathbb{H}$, определяемый формулами

$$
(\pi \mathbf{x})(0)=\mathbf{x}(0), \quad(\pi \mathbf{x})(\vartheta)=d \mathbf{x}(\vartheta) / d \vartheta, \quad \vartheta \in[-r, 0), \quad \mathbf{x} \in \mathbb{H}^{1}
$$


В пространстве $\mathbb{H}$ имеем задачу оптимальной стабилизации для дифференциального уравнения [8]

$$
\frac{d \mathbf{z}_{t}}{d t}=\hat{\mathbf{A}} \mathbf{z}_{t}+\hat{\mathbf{B}} u, \quad t \in \mathbb{R}^{+}
$$

с показателем качества

$$
\mathbf{J}=\int_{0}^{+\infty}\left(\left\langle\mathbf{C}_{x} \mathbf{z}_{t}, \mathbf{z}_{t}\right\rangle_{H}+u^{\top}(t) C_{u} u(t)\right) d t
$$

Здесь неограниченный оператор $\hat{\mathbf{A}}$ задается формулами

$$
(\hat{\mathbf{A}} \mathbf{z})(\vartheta)=\frac{d \mathbf{z}(\vartheta)}{d \vartheta}, \quad \vartheta \in[-r, 0), \quad(\hat{\mathbf{A}} \mathbf{z})(0)=\int_{-r}^{-0}[d \hat{\mu}(s)] \mathbf{z}(s)-\eta(-r) \mathbf{z}(0)
$$

и имеет область определения

$$
\begin{gathered}
\mathfrak{D}(\hat{\mathbf{A}})=\left\{\mathbf{z} \in \mathbb{H}^{1}: \mathbf{z}(-0)=\int_{-r}^{-0}[d \hat{\mu}(s)] \mathbf{z}(s)-\eta(-r) \mathbf{z}(0)\right\}, \\
\hat{\mu}(\vartheta)=\mu(\vartheta)+\eta(-r) \vartheta+\int_{\vartheta}^{0} \eta(s) d s, \quad \vartheta \in[-r, 0] ;
\end{gathered}
$$

ограниченный оператор $\hat{\mathbf{B}}: \mathbb{R}^{m} \rightarrow \mathbb{H}$ описывается формулами $(\hat{\mathbf{B}} u)(\vartheta)=0, \vartheta \in[-r, 0)$, $(\hat{\mathbf{B}} u)(0)=B u$, а ограниченный самосопряженный оператор $\mathbf{C}_{x}: \mathbb{H} \rightarrow \mathbb{H}-$

$$
\left(\mathbf{C}_{x} \mathbf{z}\right)(\vartheta)=0, \quad \vartheta \in[-r, 0), \quad\left(\mathbf{C}_{x} \mathbf{z}\right)(0)=C_{x} \mathbf{z}(0) .
$$

Оптимальное стабилизирующее управление определяется формулой

$$
\hat{\mathbf{u}}^{0}[\mathbf{z}]=-C_{u}^{-1} B^{\top}(\hat{\mathbf{U}} \mathbf{z})(0), \quad \mathbf{z} \in \mathbb{H},
$$

в которой $\hat{\mathbf{U}}$ удовлетворяет операторному уравнению Риккати [8]

$$
\hat{\mathbf{U}} \hat{\mathbf{A}}+\hat{\mathbf{A}}^{*} \hat{\mathbf{U}}+\mathbf{C}_{x}-\hat{\mathbf{U}} \mathbf{D} \hat{\mathbf{U}}=0,
$$

где ограниченный самосопряженный оператор $\mathbf{D}: \mathbb{H} \rightarrow \mathbb{H}$ задается следующим образом:

$$
(\mathbf{D} z)(\vartheta)=0, \quad \vartheta \in[-r, 0), \quad(\mathbf{D} z)(0)=D \mathbf{z}(0), \quad D=B C_{u}^{-1} B^{\top} .
$$

При нахождении решения операторного уравнения Риккати используется форма его представления

$$
(\hat{\mathbf{U}} \mathbf{z})(\vartheta)=K(\vartheta, 0) \mathbf{z}(0)+\int_{-r}^{0} K(\vartheta, s) \mathbf{z}(s) d s, \quad \vartheta \in[-r, 0], \quad \mathbf{z} \in \mathbb{H},
$$

предложенная в работе [8]. Представление (1.5) оператора $\hat{\mathbf{U}}$ определяет решение операторного уравнения Риккати (1.4), если его коэффициенты удовлетворяют системе уравнений [8]

$$
\begin{gathered}
\frac{\partial \hat{K}(\vartheta, s)}{\partial \vartheta}+\frac{\partial \hat{K}^{\top}(s, \vartheta)}{\partial s}+K(\vartheta, 0) D K(0, s)=0, \quad \vartheta, s \in[-r, 0), \\
\frac{\partial \hat{K}(\vartheta, 0)}{\partial \vartheta}+K(\vartheta, 0) D K(0,0)+(K(\vartheta, 0)+K(\vartheta,-0)) \eta(-r)=0, \quad \vartheta \in[-r, 0),
\end{gathered}
$$




$$
\begin{gathered}
(K(0,0)+K(0,-0)) \eta(-r)+\eta^{\top}(-r)(K(0,0)+K(-0,0))+K(0,0) D K(0,0)-C_{x}=0, \\
K(-r, s)=0, \quad s \in[-r, 0],
\end{gathered}
$$

где $\hat{K}(\vartheta, s)=K(\vartheta, s)-\hat{\mu}^{\top}(\vartheta)(K(0, s)+K(-0, s)), \quad \vartheta \in[-r, 0), \quad s \in[-r, 0]$.

Зная решение определяющей системы уравнений (1.6)-(1.9), оптимальное стабилизирующее управление для уравнения (1.3) можно определить формулой

$$
\hat{\mathbf{u}}^{0}[\mathbf{z}]=-C_{u}^{-1} B^{\top}\left(K(0,0) \mathbf{z}(0)+\int_{-r}^{0} K(0, s) \mathbf{z}(s) d s\right), \quad \mathbf{z} \in \mathbb{H} .
$$

Оптимальное стабилизирующее управление для системы (1.1) вычисляется как

$$
\mathbf{u}^{0}[\mathbf{x}]=-C_{u}^{-1} B^{\top}\left(K(0,0) \mathbf{x}(0)+\int_{-r}^{0} K(0, s) \frac{d \mathbf{x}(s)}{d s} d s\right), \quad \mathbf{x} \in \mathbb{H}^{1} .
$$

\section{2. Обратная задача оптимальной стабилизации}

Нахождение решения определяющей системы уравнений (1.6)-(1.9) является достаточно сложной задачей. В работе [8] показано, что ее можно свести к нахождению решения краевой задачи для специального матричного функционально-дифференциального уравнения нейтрального типа. Аналитическая сложность проблемы интегрируемости полученной краевой задачи привела к постановке обратной задачи:

Для заданного представления позиционного управления найти такие коэффициенты системы (1.1), для которых заданное представление позиционного управления определяло оптимальное стабилизирующее управление этой системы.

Для систем дифференциальных уравнений запаздывающего типа аналогичная задача решалась в [5].

Поставленная выше задача в данной статье рассматривается для следующей системы дифференциальных уравнений с последействием нейтрального типа:

$$
\frac{d x(t)}{d t}=\int_{-r}^{0}[d \eta(\vartheta)] x(t+\vartheta)+A_{0} \frac{d x(t-r)}{d t}+\int_{-r}^{0} \tilde{A}(\vartheta) \frac{d x(t+\vartheta)}{d \vartheta} d \vartheta+B u .
$$

Здесь $\mu(\vartheta)=\int_{0}^{\vartheta} \tilde{A}(s) d s, \vartheta \in(-r, 0], \mu(-r)=-\int_{-r}^{0} \tilde{A}(s) d s-A_{0}$.

В рамках обратной задачи согласно формуле (1.10) закон управления определяется заданием положительно определенной матрицы $K_{0}=K(0,0)$ и матричнозначной функции

$\Psi(\cdot) \in \mathbb{W}_{2}^{1}\left([-r, 0], \mathbb{R}^{n \times n}\right), \quad \Psi(\vartheta)=K(\vartheta, 0), \vartheta \in(-r, 0), \quad \Psi(-r)=K(-r+0,0), \quad \Psi(0)=K(-0,0)$.

Требуется найти матричнозначную функцию с ограниченной вариацией $\eta$, матричнозначную функцию $\tilde{A}(\cdot) \in \mathbb{W}_{2}^{1}\left([-r, 0], \mathbb{R}^{n \times n}\right)$ и матрицу $A_{0}$.

Для решения поставленной задачи используется определяющая система уравнений (1.6)-(1.9). Эта система записана для управляемого объекта (1.3), при описании которого используется функция с ограниченной вариацией $\hat{\mu}$. Выделяя дискретную и абсолютно непрерывные составляющие функции $\hat{\mu}_{d}, \hat{\mu}_{a}$, находим

$$
\hat{\mu}_{a}(\vartheta)=\int_{0}^{\vartheta} A(s) d s, \quad \vartheta \in[-r, 0], \quad \hat{\mu}_{d}(\vartheta)=0, \quad \vartheta \in(-r, 0], \quad \hat{\mu}_{d}(-r)=-A_{0},
$$




$$
A(\vartheta)=\tilde{A}(\vartheta)-\eta(\vartheta)+\eta(-r), \quad \vartheta \in(-r, 0) .
$$

Следовательно, поставленная обратная задача имеет не единственное решение и допускает свободу в выборе матричнозначной функции $\eta$. Будем фиксировать эту функцию и при решении обратной задачи искать матрицу $A_{0}$ и матричнозначную функцию $A(\cdot) \in \mathbb{W}_{2}^{1}\left([-r, 0], \mathbb{R}^{n \times n}\right)$.

Обратная задача решается при условии выполнения равенства

$$
K_{0} D K_{0}=C_{x}
$$

определяющего специальный выбор положительно определенной матрицы $K_{0}=K(0,0)$. Произвол в выборе $\eta$ ограничивается равенством

$$
\eta^{-1 \top}(-r)\left(K_{0}+\Psi^{\top}(0)\right)+\left(K_{0}+\Psi(0)\right) \eta^{-1}(-r)=0 .
$$

Сформулируем следующее утверждение.

Теорема 1. Пусть выполняются условия (2.2), (2.3) u $\operatorname{det}\left(K_{0}+\Psi(0)\right) \neq 0$. Тогда в решении обратной задачи матрица $A_{0}$ определяется формулой

$$
A_{0}=\left(K_{0}+\Psi^{\top}(0)\right)^{-1} \Psi^{\top}(-r),
$$

а матричнозначная функиия $A(\cdot)$ удовлетворяет линейному интегральному уравнению

$$
\begin{gathered}
\eta^{-1 \top}(-r)\left(K_{0}+\Psi^{\top}(0)\right) A(\vartheta)-A^{\top}(-\vartheta-r)\left(K_{0}+\Psi(0)\right) \eta^{-1}(-r) A_{0} \\
-\int_{-r}^{\vartheta}\left(A^{\top}(\tau-\vartheta) \hat{\Psi}^{\top}(\tau)+\hat{\Psi}(\tau-\vartheta) A(\tau)\right) d \tau=G(\vartheta),-r \leq \vartheta \leq 0 .
\end{gathered}
$$

Здесъ $\hat{\Psi}(\vartheta)=\left(\Psi^{\prime}(\vartheta)+\Psi(\vartheta) D K_{0}\right) \eta^{-1}(-r), G(\vartheta)=\Psi^{\top}(\vartheta)+\hat{\Psi}^{\top}(\vartheta)-\hat{\Psi}(-\vartheta-r) A_{0}-\int_{-r}^{\vartheta} \Psi(\tau-$ $\vartheta) D \Psi^{\top}(\tau) d \tau,-r \leq \vartheta \leq 0$.

Д о к а з а т е л ь с т в о. Преобразуем определяющую систему уравнений (1.6)-(1.9), учитывая формулы

$$
\begin{gathered}
\hat{K}(\vartheta, s)=K(\vartheta, s)-\hat{\mu}_{a}^{\top}(\vartheta)\left(\Psi^{\top}(s)+K(-0, s)\right), \quad \vartheta \in(-r, 0), \\
\hat{K}(-r, s)=-\left(\hat{\mu}_{a}^{\top}(-r)+\hat{\mu}_{d}^{\top}(-r)\right)\left(\Psi^{\top}(s)+K(-0, s)\right), \quad s \in[-r, 0) .
\end{gathered}
$$

Имеем

$$
\begin{gathered}
\frac{\partial K(\vartheta, s)}{\partial \vartheta}+\frac{\partial K^{\top}(s, \vartheta)}{\partial s}+\Psi(\vartheta) D \Psi^{\top}(s)-(\Psi(\vartheta)+K(\vartheta,-0)) A(s) \\
-A^{\top}(\vartheta)\left(\Psi^{\top}(s)+K^{\top}(s,-0)\right)=0, \quad \vartheta, s \in(-r, 0), \\
\frac{d \Psi(\vartheta)}{d \vartheta}+\Psi(\vartheta) D K_{0}+(\Psi(\vartheta)+K(\vartheta,-0)) \eta(-r)-A^{\top}(\vartheta)\left(K_{0}+\Psi(0)\right)=0, \quad \vartheta \in(-r, 0), \\
\left(K_{0}+\Psi^{\top}(0)\right) \eta(-r)+\eta^{\top}(-r)\left(K_{0}+\Psi(0)\right)+K_{0} D K_{0}-C_{x}=0, \\
K(-r+0, s)=-\hat{\mu}_{d}^{\top}(-r)\left(K^{\top}(0, s)+K(-0, s)\right), \quad s \in[-r, 0] .
\end{gathered}
$$

При $s=0$ из $(2.9)$ имеем $\Psi(-r)=A_{0}^{\top}\left(K_{0}+\Psi(0)\right)$. Откуда следует формула (2.4). 
Используя методы интегрирования дифференциальных уравнений в частных производных первого порядка и равенство (2.9), находим решение уравнения (2.6)

$$
K(\vartheta, s)=-Z(\vartheta-s-r) \hat{\mu}_{d}(-r)-\int_{-r}^{s} F(\tau+\vartheta-s, \tau) d \tau, \quad-r<\vartheta \leq s<0 .
$$

Здесь $Z(\vartheta)=\Psi(\vartheta)+K(\vartheta,-0), F(\vartheta, s)=-A^{\top}(\vartheta) Z^{\top}(s)-Z(\vartheta) A(s)+\Psi(\vartheta) D \Psi^{\top}(s),-r<\vartheta \leq$ $s<0$. Используя $(2.10)$, находим

$$
Z^{\top}(\vartheta)=\Psi^{\top}(\vartheta)-Z(-\vartheta-r) \hat{\mu}_{d}(-r)-\int_{-r}^{\vartheta} F(\tau-\vartheta, \tau) d \tau, \quad-r<\vartheta<0 .
$$

Из уравнения (2.7) выводим

$$
Z(\vartheta)=A^{\top}(\vartheta)\left(K_{0}+\Psi(0)\right) \eta^{-1}(-r)-\hat{\Psi}(\vartheta), \quad-r<\vartheta<0 .
$$

Подставляя (2.12) в (2.11), получим нелинейное интегральное уравнение

$$
\begin{gathered}
\eta^{-1 \top}(-r)\left(K_{0}+\Psi^{\top}(0)\right) A(\vartheta)-A^{\top}(-\vartheta-r)\left(K_{0}+\Psi(0)\right) \eta^{-1}(-r) A_{0} \\
+\int_{-r}^{\vartheta} A^{\top}(\tau-\vartheta)\left(\eta^{-1 \top}(-r)\left(K_{0}+\Psi^{\top}(0)\right)+\left(K_{0}+\Psi(0)\right) \eta^{-1}(-r)\right) A(\tau) d \tau \\
-\int_{-r}^{\vartheta}\left(A^{\top}(\tau-\vartheta) \hat{\Psi}^{\top}(\tau)+\hat{\Psi}(\tau-\vartheta) A(\tau)\right) d \tau=G(\vartheta), \quad-r<\vartheta<0 .
\end{gathered}
$$

При выполнении условия (2.3) зануляется нелинейное слагаемое в уравнении (2.13) и оно совпадает с линейным интегральным уравнением (2.5). Из (2.3) и (2.8) следует необходимость выполнения условия (2.2).

\section{3. Точные решения обратной задачи оптимальной стабилизации}

Точные аналитические решения уравнения (2.5) удается построить, определяя $\Psi(\cdot)$ в форме матричного квазиполинома

$$
\Psi(\vartheta)=\sum_{k=0}^{M} \sum_{j=0}^{M_{k}}(\vartheta+r)^{j} e^{\lambda_{k}(\vartheta+r)} \Psi_{k j}, \quad \vartheta \in[-r, 0],
$$

где $\Psi_{k j}$ - матрицы размерности $n \times n, j=\overline{0, M_{k}}, k=\overline{0, M}, \lambda_{0}=0$. Если $\lambda_{k}$ - вещественное число, то матрицы $\Psi_{k j}$ имеют вещественные элементы, а каждому невещественному числу $\lambda_{k}$ соответствует сопряженное число $\lambda_{k^{\prime}}=\overline{\lambda_{k}}$ и $\Psi_{k^{\prime} j}=\overline{\Psi_{k j}}$. Последние условия гарантируют вещественность матричной функции $\Psi$. Матричнозначные функции $\hat{\Psi}$ и $G$ являются квазиполиномами. Имеет место формула

$$
\hat{\Psi}(\vartheta)=\sum_{k=0}^{M} \sum_{j=0}^{M_{k}}(\vartheta+r)^{j} e^{\lambda_{k}(\vartheta+r)} \hat{\Psi}_{k j}, \quad \vartheta \in[-r, 0],
$$

где $\hat{\Psi}_{k j}$ - известные матрицы размерности $n \times n, j=\overline{0, M_{k}}, k=\overline{0, M}$. 
Определим функции

$$
\begin{gathered}
X_{k j}(\vartheta)=\int_{-r-\vartheta}^{0}(z+r+\vartheta)^{j} e^{\lambda_{k}(z+r+\vartheta)} A^{\top}(z) d z, \\
Y_{k j}(\vartheta)=\int_{-r}^{\vartheta}(z+r-\vartheta)^{j} e^{\lambda_{k}(z+r-\vartheta)} A(z) d z, \quad j=\overline{0, M_{k}}, \quad k=\overline{0, M}, \quad \vartheta \in[-r, 0] .
\end{gathered}
$$

Введем обозначения $X_{1 k j}(\vartheta)=X_{k j}(\vartheta), X_{2 k j}(\vartheta)=X_{k j}^{\top}(-r-\vartheta), Y_{1 k j}(\vartheta)=Y_{k j}(\vartheta), Y_{2 k j}(\vartheta)=$ $Y_{k j}^{\top}(-r-\vartheta), j=\overline{0, M_{k}}, k=\overline{0, M}, G_{1}(\vartheta)=G(\vartheta), G_{2}(\vartheta)=G^{\top}(-r-\vartheta), A_{1}(\vartheta)=A(\vartheta), A_{2}(\vartheta)=$ $A^{\top}(-r-\vartheta), \vartheta \in[-r / 2,0]$.

Лемма. Функиии $X_{1 k j}(\cdot), X_{2 k j}(\cdot), Y_{1 k j}(\cdot), Y_{2 k j}(\cdot), j=\overline{0, M_{k}}, k=\overline{0, M}$, являются компонентами решения следующей краевой задачи для обыкновенных дифференииальных уравнений

$$
\begin{gathered}
X_{1 k 0}^{\prime}=\lambda_{k} X_{1 k 0}+A_{2}(\vartheta), \quad Y_{1 k 0}^{\prime}=-\lambda_{k} Y_{1 k 0}+e^{\lambda_{k} r} A_{1}(\vartheta), \\
X_{2 k 0}^{\prime}=-\lambda_{k} X_{2 k 0}-A_{1}(\vartheta), \quad Y_{2 k 0}^{\prime}=\lambda_{k} Y_{2 k 0}-e^{\lambda_{k} r} A_{2}(\vartheta), \\
X_{1 k j}^{\prime}=\lambda_{k} X_{1 k j}+j X_{1 k(j-1)}, \quad Y_{1 k j}^{\prime}=-\lambda_{k} Y_{1 k j}-j Y_{1 k(j-1)}+r^{j} e^{\lambda_{k} r} A_{1}(\vartheta), \\
X_{2 k j}^{\prime}=-\lambda_{k} X_{2 k j}-j X_{2 k(j-1)}, \quad Y_{2 k j}^{\prime}=\lambda_{k} Y_{2 k j}+j Y_{2 k(j-1)}+r^{j} e^{\lambda_{k} r} A_{2}(\vartheta), \\
j=\overline{1, M_{k}}, \quad k=\overline{0, M}, \\
X_{2 k j}(0)=0, Y_{2 k j}(0)=0, \quad X_{1 k j}(-r / 2)=X_{2 k j}^{\top}(-r / 2), \\
Y_{1 k j}(-r / 2)=Y_{2 k j}^{\top}(-r / 2), \quad j=\overline{0, M_{k}}, k=\overline{0, M} .
\end{gathered}
$$

Д о к а з а т е л ь с т в о. Используя определение функций $X_{k j}, Y_{k j}, j=\overline{0, M_{k}}, k=\overline{0, M}$, находим, что они являются компонентами решения следующей краевой задачи для дифференциальных уравнений:

$$
\begin{gathered}
X_{k 0}^{\prime}(\vartheta)=\lambda_{k} X_{k 0}(\vartheta)+A^{\top}(-r-\vartheta), \quad Y_{k 0}^{\prime}(\vartheta)=-\lambda_{k} Y_{k 0}(\vartheta)+e^{\lambda_{k} r} A(\vartheta), \\
X_{k j}^{\prime}(\vartheta)=\lambda_{k} X_{k j}(\vartheta)+j X_{k(j-1)}(\vartheta), \quad Y_{k j}^{\prime}(\vartheta)=-\lambda_{k} Y_{k j}(\vartheta)-j Y_{k(j-1)}(\vartheta)+r^{j} e^{\lambda_{k} r} A(\vartheta), \\
j=\overline{0, M_{k}}, \quad k=\overline{0, M}, \\
X_{k j}(-r)=0, Y_{k j}(-r)=0, \quad j=\overline{0, M_{k}}, k=\overline{0, M} .
\end{gathered}
$$

Используя определение функций $X_{1 k j}(\cdot), X_{2 k j}(\cdot), Y_{1 k j}(\cdot), Y_{2 k j}(\cdot), j=\overline{0, M_{k}}, k=\overline{0, M}, A_{1}(\cdot), A_{2}(\cdot)$, преобразуем краевую задачу (3.2) в краевую задачу (3.1).

Рассмотрим алгебраические уравнения

$$
\begin{gathered}
J^{\top} A_{1}(\vartheta)-A_{2}(\vartheta) \hat{A}_{0}-\sum_{k=0}^{M} \sum_{j=0}^{M_{k}}\left(X_{1 k j}(\vartheta) \hat{\Psi}_{k j}^{\top}+\hat{\Psi}_{k j} Y_{1 k j}(\vartheta)\right)=G_{1}(\vartheta), \\
-\hat{A}_{0}^{\top} A_{1}(\vartheta)+A_{2}(\vartheta) J-\sum_{k=0}^{M} \sum_{j=0}^{M_{k}}\left(Y_{2 k j}(\vartheta) \hat{\Psi}_{k j}^{\top}+\hat{\Psi}_{k j} X_{2 k j}(\vartheta)\right)=G_{2}(\vartheta), \\
\vartheta \in[-r / 2,0], \quad J=\left(K_{0}+\Psi(0)\right) \eta^{-1}(-r), \quad \hat{A}_{0}=J A_{0} .
\end{gathered}
$$


Теорема 2. Пусть выполнены условия теоремы 1. Тогда решение линейного интегрального уравнения определяется формулами $A(\vartheta)=A_{1}(\vartheta)$ при $\vartheta \in[-r / 2,0], A(\vartheta)=A_{2}^{\top}(-r-\vartheta)$ при $\vartheta \in[-r,-r / 2)$. Здесь $A_{1}(\cdot)$ и $A_{2}(\cdot)$ являются компонентами решения краевой задачи для гибридной системы обыкновенных дибференциалъных уравнений (3.1) и алгебраических уравнений (3.3).

Д о к а з а т е л ь с т в о. Учитывая введенные обозначения и определение функции $\hat{\Psi}$ из уравнения (2.5), получим

$$
J^{\top} A(\vartheta)-A(-r-\vartheta)^{\top} \hat{A}_{0}-\sum_{k=0}^{M} \sum_{j=0}^{M_{k}}\left(X_{k j}(\vartheta) \hat{\Psi}_{k j}^{\top}+\hat{\Psi}_{k j} Y_{k j}(\vartheta)\right)=G(\vartheta), \quad \vartheta \in[-r, 0] .
$$

Разбивая отрезок $[-r, 0]$ на два отрезка и на отрезке $[-r,-r / 2]$ производя замену переменных $\vartheta \rightarrow-r-\vartheta$, получим систему алгебраических уравнений (3.3). Система обыкновенных дифференциальных уравнений (3.1) и алгебраических уравнений (3.3) является замкнутой.

\section{4. Пример}

При решении обратной задачи оптимальной стабилизации для системы (2.1) полагаем $n=2, m=1, r=1, B=(1,0)^{\top}, C_{u}=1, C_{x}=\left(\begin{array}{ll}1 & 0 \\ 0 & 0\end{array}\right)$. Тогда матрица $D=B C_{u}^{-1} B^{\top}=\left(\begin{array}{ll}1 & 0 \\ 0 & 0\end{array}\right)$ и условие (2.2) выполняется для матрицы $K_{0}=I_{2}$.

Найдем решение обратной задачи для матричной функции $\Psi(\vartheta)=\Psi=$ const, $\vartheta \in[-1,0]$, где $\Psi=I_{2}$. Полагаем $\eta(\vartheta)=O_{2}, \vartheta \in(-1,0]$, и из условия $(2.3)$ находим матрицу $\eta(-1)=$ $\left(\begin{array}{cc}0 & 2 \\ -2 & 0\end{array}\right)$.

Определяем матричные функции $\hat{\Psi}(\vartheta)=\hat{\Psi}=$ const, $G(\vartheta)=\Gamma_{0}-\Gamma_{1}(\vartheta+1), \vartheta \in[-1,0]$, где $\hat{\Psi}=\left(\begin{array}{cc}0 & -1 / 2 \\ 0 & 0\end{array}\right), \Gamma_{0}=\left(\begin{array}{cc}1 & 1 / 4 \\ -1 / 2 & 1\end{array}\right), \Gamma_{1}=\left(\begin{array}{ll}1 & 0 \\ 0 & 0\end{array}\right)$.

Запишем алгебраические уравнения (3.3) для данного примера:

$$
\begin{gathered}
J^{\top} A_{1}(\vartheta)-A_{2}(\vartheta) \hat{A}_{0}-\left(X_{100}(\vartheta) \hat{\Psi}^{\top}+\hat{\Psi} Y_{100}(\vartheta)\right)=G_{1}(\vartheta), \\
-\hat{A}_{0}^{\top} A_{1}(\vartheta)+A_{2}(\vartheta) J-\left(Y_{200}(\vartheta) \hat{\Psi}^{\top}+\hat{\Psi} X_{200}(\vartheta)\right)=G_{2}(\vartheta), \quad \vartheta \in[-1 / 2,0],
\end{gathered}
$$

в которых $\hat{A}_{0}=\frac{1}{2} J, J=\left(\begin{array}{cc}0 & -1 \\ 1 & 0\end{array}\right)$, функции $X_{100}(\cdot), Y_{100}(\cdot), X_{200}(\cdot), Y_{200}(\cdot)$ являются компонентами решений системы обыкновеннных дифференциальных уравнений

с краевыми условиями

$$
\begin{gathered}
\frac{d X_{100}}{d \vartheta}=A_{2}(\vartheta), \quad \frac{d Y_{100}}{d \vartheta}=A_{1}(\vartheta), \\
\frac{d X_{200}}{d \vartheta}=-A_{1}(\vartheta), \quad \frac{d Y_{200}}{d \vartheta}=-A_{2}(\vartheta),
\end{gathered}
$$

$$
\begin{gathered}
X_{200}(0)=Y_{200}(0)=0, \quad X_{100}(-1 / 2)=X_{200}^{\top}(-1 / 2), \\
Y_{100}(-1 / 2)=Y_{200}^{\top}(-1 / 2) .
\end{gathered}
$$

Исключая в $(3.3)$ функции $X_{100}(\cdot), Y_{100}(\cdot), X_{200}(\cdot), Y_{200}(\cdot)$, получим систему обыкновенных дифференциальных уравнений с постоянными коэффициентами

$$
J^{\top} \frac{d A_{1}}{d \vartheta}-\frac{d A_{2}}{d \vartheta} \hat{A}_{0}=A_{2} \hat{\Psi}^{\top}+\hat{\Psi} A_{1}-\Gamma_{1},
$$




$$
-\hat{A}_{0}^{\top} \frac{d A_{1}}{d \vartheta}+\frac{d A_{2}}{d \vartheta} J=-A_{2} \hat{\Psi}^{\top}-\hat{\Psi} A_{1}+\Gamma_{1}^{\top}
$$

и краевыми условиями

$$
\begin{aligned}
& J^{\top} A_{1}(-1 / 2)-A_{2}(-1 / 2) \hat{A}_{0}-\left(X_{100}(-1 / 2) \hat{\Psi}^{\top}+\hat{\Psi} Y_{100}(-1 / 2)\right)=G_{1}(-1 / 2), \\
& -\hat{A}_{0}^{\top} A_{1}(-1 / 2)+A_{2}(-1 / 2) J-\left(Y_{200}(-1 / 2) \hat{\Psi}^{\top}+\hat{\Psi} X_{200}(-1 / 2)\right)=G_{2}(-1 / 2) .
\end{aligned}
$$

Используя краевые условия (4.1) и равенство $A_{2}(-1 / 2)=A_{1}^{\top}(-1 / 2)$, убеждаемся в эквивалентности краевых условий (4.3) и (4.4). Используя определения функций $X_{200}(\cdot)$ и $Y_{200}(\cdot)$, находим $X_{200}(-1 / 2)=\int_{-1 / 2}^{0} A_{1}(s) d s$ и $Y_{200}(-1 / 2)=\int_{-1 / 2}^{0} A_{2}(s) d s$. Для решений системы $(4.2)$ имеет место равенство

$\left(J-\hat{A}_{0}\right)^{\top} A_{1}(\vartheta)+A_{2}(\vartheta)\left(J-\hat{A}_{0}\right)=C=\left(J-\hat{A}_{0}\right)^{\top} A_{1}(-1 / 2)+A_{1}^{\top}(-1 / 2)\left(J-\hat{A}_{0}\right), \quad \vartheta \in[-1 / 2,0]$,

с помощью которого исходную задачу можно свести к нахождению решения следующей краевой задачи

$$
\begin{gathered}
J^{\top} \frac{d A_{1}}{d \vartheta}+\left(J-\hat{A}_{0}\right)^{\top} \frac{d A_{1}}{d \vartheta}\left(J-\hat{A}_{0}\right)^{-1} \hat{A}_{0} \\
=-\left(J-\hat{A}_{0}\right)^{\top} A_{1}\left(J-\hat{A}_{0}\right)^{-1} \hat{\Psi}^{\top}+\hat{\Psi} A_{1}+C\left(J-\hat{A}_{0}\right)^{-1} \hat{\Psi}^{\top}-\Gamma_{1}, \\
\hat{A}_{0}^{\top} A_{1}(0)+\left(J-\hat{A}_{0}\right)^{\top} A_{1}(0)\left(J-\hat{A}_{0}\right)^{-1} J=C\left(J-\hat{A}_{0}\right)^{-1} J-\Gamma_{0}^{\top} .
\end{gathered}
$$

Матричное решение краевой задачи (4.5), (4.6) определяется формулой

$$
A_{1}(\vartheta)=\left(\begin{array}{cc}
1 / 2 & -2 \\
-\vartheta & 0
\end{array}\right), \quad \vartheta \in[-1 / 2,0]
$$

Используя теорему 2 и полученную в примере связь функций $A_{1}$ и $A_{2}$, а также формулу $\widetilde{A}(\vartheta)=A(\vartheta)-\eta(-1), \vartheta \in[-1,0]$, находим матричнозначную функцию

$$
\tilde{A}(\vartheta)=\left(\begin{array}{cc}
1 / 2 & -4 \\
-\vartheta+2 & 0
\end{array}\right) \in[-1,0] .
$$

Используя формулу (2.4), находим матрицу $A_{0}=\frac{1}{2} I_{2}$.

\section{Заключение}

Нахождение оптимального стабилизирующего управления для автономной линейной системы дифференциальных уравнений с последействием нейтрального типа связано с решением нелинейной краевой задачи для функционально-дифференциального уравнения. Для обратной задачи стабилизации требуется определять решение линейного интегрального уравнения. В работе приведены условия, при выполнении которых процедура нахождения решения обратной задачи стабилизации для автономной линейной системы дифференциальных уравнений с последействием нейтрального типа непосредственно связана с интегрированием краевой задачи для автономной системы обыкновенных дифференциальных уравнений.

\section{СПИСОК ЛИТЕРАТУРЫ}

1. Красовский Н.Н. Об аналитическом конструировании оптимального регулятора в системе с запаздываниями времени // Прикладная математика и механика. 1962. Т. 26, вып. 1. С. 39-51.

2. Gibson J.S. Linear-quadratic optimal control of hereditary differential systems: infinite dimensional Riccati equations and numerical approximations // SIAM J. Control Optim. 1983. Vol. 21, no. 5. P. 95135. doi: $10.1137 / 0321006$. 
3. Delfour M.C., McCalla C., Mitter S.K. Stability and the infinite-time quadratic cost problem for linear hereditary differential systems // SIAM J. Control. 1975. Vol. 13, no. 1. P. 48-88. doi: $10.1137 / 0313004$.

4. Андреева Е.А., Колмановский В.Б., Шайхет Л.Е. Управление системами с последействием. Москва: Наука, 1992. 336 с.

5. Долгий Ю.Ф. К стабилизации линейных автономных систем дифференциальных уравнений с распределенным запаздыванием // Автоматика и телемеханика. 2007. № 10. С. 92-105.

6. Желонкина Н.И., Ложников А.Б., Сесекин А.Н. Об оптимальной стабилизации импульсным управлением линейных систем с последействием // Автоматика и телемеханика. 2013. № 11. C. $39-48$.

7. Yanushevsky R.T. Optimal control of linear differential-difference systems of neutral type// Int. J. Control. 1989. Vol. 49, no. 6. P. 1835-1850.

8. Долгий Ю.Ф. Оптимальная стабилизация систем дифференциальных уравнений с последействием нейтрального типа // Тр. Междунар. конф. "Динамика систем и процессы управления". 2015. C. $155-162$.

Поступила 6.11.2018

После доработки 8.02.2019

Долгий Юрий Филиппович

Принята к публикации 11.02.2019

д-р физ.-мат. наук, профессор

ведущий науч. сотрудник

Институт математики и механики им. Н. Н. Красовского УрО РАН;

Уральский федеральный университет

г. Екатеринбург

e-mail: yurii.dolgii@imm.uran.ru

\section{REFERENCES}

1. Krasovskii N.N. On the analytic construction of an optimal control in a system with time lags. PMM, J. Appl. Math. Mech., 1962, vol. 26, no. 1, pp. 50-67. doi: 10.1016/0021-8928(62)90101-6.

2. Gibson J.S. Linear-quadratic optimal control of hereditary differential systems: infinite dimensional Riccati equations and numerical approximations. SIAM J. Control Optim., 1983, vol. 21, no. 1, pp. 95139. doi: $10.1137 / 0321006$.

3. Delfour M.C., McCalla C., Mitter S.K. Stability and the infinite-time quadratic cost problem for linear hereditary differential systems. SIAM J. Control, 1975, vol. 13, no. 1, pp. 48-88. doi: 10.1137/0313004.

4. Andreeva E.A., Kolmanovskii V.B., Shaikhet L.E. Upravlenie sistemami s posledeistviem (Control of systems with aftereffect). Moscow: Nauka Publ., 1992, 336 p. ISBN: 5-02-014875-X.

5. Dolgii Yu.F. Stabilization of linear autonomous systems of differential equations with distributed delay. Autom. Remote Control, 2007, vol. 68, no. 10, pp. 1813-1825. doi: 10.1134/S0005117907100098.

6. Zhelonkina N.I., Lozhnikov A.B., Sesekin A.N. On pulse optimal control of linear systems with aftereffect. Autom. Remote Control, 2013, vol. 74, no. 11, pp. 1802-1809. doi: 10.1134/S0005117913110039.

7. Yanushevsky R.T. Optimal control of linear differential-difference systems of neutral type. Int. J. Control, 1989, vol. 49, no. 6, pp. 1835-1850.

8. Dolgii Yu.F. Optimal stabilization of systems of differential equations with aftereffect of neutral type. Proc. Systems Dynamics and Control Processes - SDCP-2014, 2015, pp. 155-162 (in Russian). ISBN: 978-5-8295-0364-2 .

Received November 6, 2018

Revised February 8, 2019

Accepted February 11, 2019

Yuriy Filippovich Dolgii, Dr. Phys.-Math. Sci., Prof., Krasovskii Institute of Mathematics and Mechanics of the Ural Branch of the Russian Academy of Sciences, Yekaterinburg, 620108 Russia; Ural Federal University, Yekateriburg, 620002, Russia, e-mail: yurii.dolgii@imm.uran.ru . 